\title{
Análisis de la Influencia de la Radiación Solar en una Planta de Ciclo Combinado con Colectores Solares Integrados mediante Simulación
}

\author{
Carolina V. Ponce y José O. Valderrama \\ Fac. de Ingeniería, Dpto. de Ingeniería Mecánica, Univ. de La Serena, Benavente 980, La Serena-Chile \\ (e-mail: cponce@userena.cl; jvalderr@userena.cl)
}

Recibido Oct. 2, 2015; Aceptado Oct. 27, 2015; Versión final Ene. 28, 2016, Publicado Jun. 2016

\begin{abstract}
Resumen
Se analiza el efecto de la variación de la radiación solar sobre algunas variables de una planta termoeléctrica de ciclo combinado (turbina de gas y turbina de vapor) con colectores solares integrados (Integrated Solar Combined Cycle, ISCC). Una planta ISCC consta de tres componentes: i) la planta termoeléctrica de ciclo combinado, ii) el campo de colectores solares distribuidos, y iii) un generador de vapor solar. En este trabajo, la planta de ciclo combinado y la de colectores solares distribuidos son integradas agregando un generador de vapor a la planta solar que produce vapor para la planta de ciclo combinado. Se resuelven los balances de materia y energía en el generador de vapor solar y se realiza la implementación computacional usando Matlab-Simulink®. Los equipos de ambas plantas, la planta termoeléctrica de ciclo combinado y la planta ISCC, son los mismos, y solo cambia la fuente de energía que calienta parte del vapor que va al sobre calentador. La principal variable de interés y que es analizada con el simulador es el flujo de vapor que se produce en el generador de vapor solar y que resulta ser de un máximo de $10 \%$ del vapor consumido en la planta total durante las horas de máxima radiación (11 am a $15 \mathrm{pm}$ ). El simulador desarrollado es una buena herramienta para estudiar los efectos de las variaciones de la radiación solar en una planta de ciclo combinado-solar.
\end{abstract}

\section{Influence of Solar Radiation on a Combined Cycle Plant with Integrated Solar Collectors by Simulation}

\begin{abstract}
The effect of the variation of daily solar radiation on some variables of a thermoelectric power plant using a combined cycle with integrated solar collectors is analyzed. A combined cycle with integrated solar collectors plant (Integrated Solar Combined Cycle, ISCC) consists of three main components: i) the combined cycle power plant, ii) the field of distributed solar collectors and iii) a solar vapor generator. In this work, the combined cycle power plant and the distributed solar collector's plant are integrated by adding a solar vapor generator to the solar plant that produces vapor for the ISCC plant. Material and energy balances are performed in the solar vapor generator and the computational implementation is done using Matlab-Simulink ${ }^{\circledR}$. The pieces of equipment of both plants, the combined cycle power plant and the ISCC plant, are the same and the only change done is the source of energy that heats part of the vapor that goes to the super heater. The main variable of interest that is analyzed using the simulator is the flow of vapor that is produced in the solar vapor generator and that contributes with a maximum of $10 \%$ of the total vapor consumed during the peak hours of solar radiation (11 am to $15 \mathrm{pm}$ ). The simulator developed in this work represents a good tool to study the effects of variation of daily solar radiation in a combined-solar plant.
\end{abstract}




\section{INTRODUCCION}

La energía juega un papel fundamental en el desarrollo social y económico de los países. El funcionamiento de las sociedades modernas depende por completo de una adecuada disponibilidad de energía, tanto para el desarrollo de sus actividades productivas como cotidianas. En este mismo sentido, un mayor desarrollo económico de una nación implica necesariamente un crecimiento en su consumo de energía. Existe una relación lineal entre el Producto Interno Bruto (PIB) de los países y el consumo de energía eléctrica (ambos per-cápita), no sólo en países desarrollados sino también en países en desarrollo. Enfrentar los requerimientos eléctricos de manera sustentable es una necesidad actual prioritaria para el desarrollo de los países (Central Energía 2010).

Como se sabe, el cambio climático global es la mayor amenaza que enfrenta la vida de hoy en día. Los científicos advierten que si aumenta la temperatura global más de $2^{\circ} \mathrm{C}$ en relación con los niveles preindustriales, estaremos generando un cambio climático catastrófico y desencadenando procesos que provocarán la liberación de más emisiones de gases de efecto invernadero (GEI) (Greenpeace, 2013). La principal causa de este fenómeno es la concentración desmedida de GEI en la atmósfera, derivada de la quema excesiva de combustibles fósiles (petróleo, carbón y gas) y de la deforestación. La situación actual de producción y consumo de energía es insostenible por dos razones básicas: los yacimientos de esos combustibles se están agotando y ocasiona graves trastornos ambientales. El Panel Intergubernamental sobre Cambio Climático de la Organización de las Naciones Unidas ha identificado un veloz aumento de las concentraciones de dióxido de carbono y otros GEI en décadas recientes. Estos gases acentúan el efecto invernadero y provocan los cambios globales conocidos (Greenpeace, 2013).

Teniendo en consideración el problema del calentamiento global, es que en los últimos años se han añadido a las fuentes de generación de energía convencionales las plantas de ciclo combinado con colectores solares integrados (ISCC por su sigla en inglés: Integrated Solar Combined Cycle). Las plantas ISCC o plantas integradas, se construyen integrando una planta solar a una planta de ciclo combinado (CC). Sin embargo, no se conoce cuál es la fracción económicamente óptima de energía que debe aportar la planta solar a la planta integrada. Desde el punto de vista termodinámico el aporte de energía solar debe ser utilizado para reemplazar la energía a la más alta temperatura posible. Pero la temperatura del fluido de transferencia de energía usada en la planta solar (usualmente un aceite) está limitada por la temperatura de descomposición de dicho fluido (Dersch, et al., 2004). Algunos autores han realizado estudios y entregan recomendaciones con respecto al porcentaje de aporte solar que debe tener una planta integrada. Kelly y Herrmann (2001) plantean que para mantener una constribución solar eficiente con el uso de estanque de almacenamiento, el aporte solar debe estar sobre el 10\%. Kelly et al. (2001) sugieren que el método más eficiente para integrar la central térmica solar a la planta de CC es sacar el agua de la caldera, desde la segunda etapa del economizador (alta temperatura), producir vapor saturado a alta presión y devolver el vapor a la caldera para ser recalentado por los gases de escape de la turbina de gas. Este es el método usado en el simulador propuesto en este trabajo.

La primera planta de generación de energía eléctrica que integra un planta de ciclo combinado con una de colectores solares distribuidos (DSC: Distributed Solar Colector), fue construida en la localidad de HassiR'mel en Argelia (Behar, et al., 2011). Ésta planta está compuesta por un generador de ciclo combinado de $300 \mathrm{MW}$ de potencia y una planta solar de $20 \mathrm{MW}$ de potencia. Esta última corresponde a un campo de colectores solares distribuidos que ocupa un terreno de aproximadamente 180 mil metros cuadrados por los que, a través de un tubo, circula un aceite térmico (heat transfer fluid, HTF) que ingresa al campo de colectores a una temperatura de $293^{\circ} \mathrm{C}$ y sale a $393^{\circ} \mathrm{C}$. Esta planta no posee estanque de almacenamiento como sugieren Kelly y Herrmann (2001). Existe otra planta de este tipo en funcionamiento que se ubica en Marruecos en la localidad de At Ain Beni Mathar (Aftzoglou, 2011). Egipto cuenta con una planta de $150 \mathrm{MW}$ de potencia totales, con un aporte solar de $20 \mathrm{MW}$ de potencia, e Irán cuenta con una planta de $467 \mathrm{MW}$ y $20 \mathrm{MW}$ de potencia de aporte solar.

Debido a que este tipo de tecnología es relativamente nueva, se han realizado diversos estudios técnicos y económicos para evaluar la factibilidad de este tipo de plantas. Hosseini et al. (2005) realizan una evaluación económica y técnica de una planta ISCC ubicada en Irán. En ese trabajo se evalúa la eficiencia térmica y el factor de capacidad de la planta, definido como la razón entre la energía anual producida y la máxima energía anual que se puede producir. Se evalúan además las consideraciones ambientales por medio de la disminución de las emisiones de $\mathrm{CO}_{2}$, la inversión económica, el costo de combustible y otros parámetros. Horn et al. (2004), también realizan un análisis económico de la planta integrada ubicada en Egipto. El estudio realizado incluye el valor actual neto de dos alternativas de tecnologías de concentración solar, los colectores solares distribuidos y la torre de concentración solar. Los autores determinan además el costo incremental de agregar una planta solar a una planta base de ciclo combinado y los costos nivelados de producción de electricidad (levelized cost) para la planta ISCC y la planta solar. En ambos estudios se 
concluye que el proyecto de construcción de una planta integrada provee beneficios ambientales y económicos para la generación de energía eléctrica en Egipto.

La aparición de plantas de ciclo combinado con colectores solares integrados trae como consecuencia la necesidad de crear herramientas adecuadas para el estudio de su comportamiento dinámico, el análisis de sus variables y el desarrollo de estrategias de control adecuadas para mejorar y optimizar su desempeño. La implementación de estrategias de control requiere disponer de un simulador dinámico, como el que se presenta en este trabajo (Ordys et al., 1994). Utilizando el simulador dinámico desarrollado se analiza el comportamiento dinámico de algunas variables de las plantas integradas, cuando varía la radiación solar. Es así como el desarrollo del simulador dinámico presentado en este trabajo es fundamental para futuros estudios dinámicos y diseño de estrategias de control de este tipo de plantas.

El diseño del simulador dinámico de una planta de ciclo combinado con colectores solares integrados se realizó a partir del simulador de una planta de ciclo combinado desarrollado por Sáez et al. (2002) y del simulador de la planta de colectores solares distribuidos ACUREX, diseñado por Camacho et al. (1993). EI simulador propuesto en este trabajo incluye los tres componentes básicos de una planta ISCC: la planta termoeléctrica de ciclo combinado, la planta de colectores solares distribuidos con estanque de almacenamiento y el generador de vapor solar. El simulador se diseña utilizando el software Matlab Simulink®. La Figura 1 muestra un esquema general de la planta integrada, donde se aprecia la interconexión de los diferentes equipos que conforman una planta ISCC. También se destaca el sobre calentador como parte de la caldera. Los componentes que forman parte de la planta solar son la planta de colectores solares, el estanque de almacenamiento, y el generador de vapor solar.

\section{DESCRIPCION DE PLANTAS DE CICLO COMBINADO CON COLECTORES SOLARES INTEGRADOS}

Como se explicó en la introducción, las plantas ISCC se construyen integrando una planta solar a una planta de ciclo combinado. La turbina de gas de la planta de CC opera usando los gases de combustión de un combustible fósil (por ejemplo gas natural). Los gases de escape de la turbina, a una temperatura relativamente alta se inyectan en la caldera para la producción de vapor. Este vapor sobrecalentado, sale de la caldera y se inyecta a una turbina de vapor, donde se aprovecha para producir electricidad. Algunas configuraciones utilizan un quemador auxiliar en la caldera. El quemador auxiliar tiene la función de aumentar la temperatura de la caldera por medio del uso de combustible, aumentando la cantidad de vapor producido. La turbina de vapor también puede ser de tres etapas, alta presión, media presión y baja presión. Este arreglo aumenta la eficiencia del sistema, ya que no se pierden los gases de escape de la turbina de gas (Ordys, et al., 1994).

Por otro lado, en la planta solar una bomba saca el aceite frio del estanque de almacenamiento, desde su parte inferior y lo lleva a los colectores solares. A medida que el aceite circula por los colectores, se calienta y es inyectado nuevamente al estanque, en su parte superior, donde se ubica el aceite caliente. Este aceite caliente es llevado hacia el generador de vapor solar (GVS) donde es aprovechado en la producción de vapor. El agua de alimentación del GVS proviene del precalentador de alta presión de la planta de ciclo combinado y el GVS produce vapor ligeramente sobre calentado. Este vapor regresa a la caldera y junto con el vapor que viene desde el sobre calentador convencional, es finalmente recalentado a la temperatura final de operación. Este vapor recalentado, sale de la caldera y se inyecta a la turbina de vapor, donde el trabajo producido se aprovecha para generar electricidad.

Como se mencionó en la introducción, el desarrollo del simulador de la planta integrada se basa en una propuesta anterior desarrollada por Sáez et al. (2002) para la planta de ciclo combinado (CC) y la propuesta por Camacho et al. (1993) para la planta solar de colectores solares distribuidos (DSC). En el nuevo simulador propuesto, desarrollado en Matlab®, la planta de CC y la DSC son integradas agregando un generador de vapor solar (GVS) a la DSC que produce vapor para la planta integrada. Los equipos de ambas plantas, la CC y la ISCC, son los mismos y solo cambia la fuente de energía que calienta parte del vapor que va al recalentador. Se diseñó además un simulador para el sobre calentador de la ISCC. La DSC tiene un sistema de control cuyo objetivo es mantener constante la temperatura de salida del aceite, manipulando el flujo de aceite que circula por los colectores. Las variaciones del flujo de aceite producen a su vez variaciones en el flujo de vapor que aporta el GVS a la planta integrada.

Un esquema que representa el proceso de intercambio de energía entre el aceite proveniente de la planta solar y el agua proveniente del economizador de la caldera de la planta de ciclo combinado, se muestra en la Figura 2. EI GVS aprovecha la energía que tiene el aceite que fue previamente calentado en la planta de colectores solares. La energía del aceite es transferida al agua líquida produciendo vapor, que es el que va a la planta de ciclo combinado. 


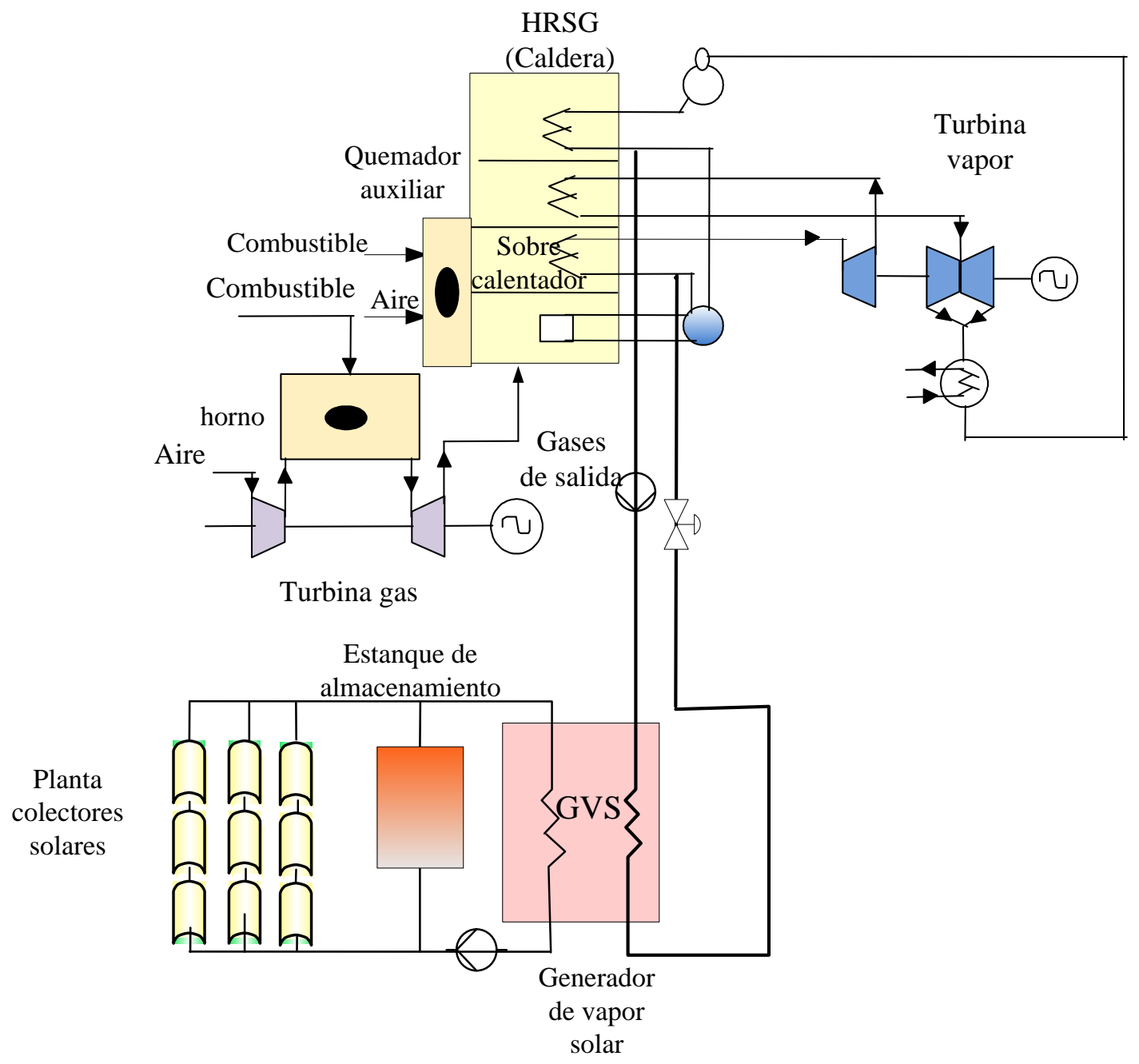

Fig. 1: Esquema de la planta de ciclo combinado con colectores solares integrados

El aceite proveniente de la planta solar posee una temperatura de entrada denominada $T_{\text {ea }}$ y un flujo másico denominado $m_{a}$. El agua líquida que entra al GVS tiene una temperatura de entrada $T_{\text {ew }}$, pero al circular por el intercambiador de calor el agua se calienta a una temperatura determinada por diseño $T_{\text {sw }}$, produciendo vapor saturado con una entalpía $h_{g m}$. Luego a la salida, se tiene un flujo de vapor $w_{g m}$.

\section{METODOLOGIA}

Para el desarrollo del simulador del generador de vapor solar, se adoptaron criterios de diseño expuestos en la literatura. Basándose en lo planteado por Dersch et al. (2004), Kelly et al. (2001) y Kelly y Herrmann (2001) se diseñó el GVS considerando que el 10\% del agua de alimentación de la planta de ciclo combinado se inyecta en el GVS. Se consideró además que el fluido de trabajo en la planta solar es aceite Therminol 55 (fluido térmico usado en aplicaciones a temperatura moderada). Un vez definidos estos criterios, se determinaron las variables relevantes del modelo y se programó el simulador en Matlab Simulink®.

\section{Definición de variables del modelo}

La Figura 3 muestra un diagrama con las variables de entrada y salida que participan en el diseño del simulador del GVS. Las variables de entrada que se utilizan en el simulador del GVS están representadas a la izquierda y las variables de salida a la derecha de la figura. En la figura, $w_{e g}$ es el flujo másico de agua líquida proveniente del economizador, $m_{a}$ es el flujo másico de aceite proveniente de la planta solar y $P_{e g}$ es la presión de entrada del agua, que viene de la salida de la bomba instalada para aumentar la presión del flujo. $T_{\text {ea }}$ es la temperatura de entrada del aceite que viene desde la planta solar. Las variables de salida son: $T_{s w}$ temperatura del vapor saturado que sale del GVS a la presión de la cañería. También, $w_{g m}$ es el flujo de vapor producido en el GVS, $h_{g m}$ es la entalpía del vapor y $Q_{g m}$ es el calor suministrado al vapor producido en el GVS. Las ecuaciones utilizadas en el diseño del simulador de GVS se muestran en la Tabla 1. En dicha tabla se muestra el balance de energía y la relación de flujo derivada de dicho balance, además de las propiedades fisicoquímicas y termodinámicas necesarias para los cálculos (calor específico del aceite, entalpía del vapor, calor de vaporización y temperatura de saturación del agua). 


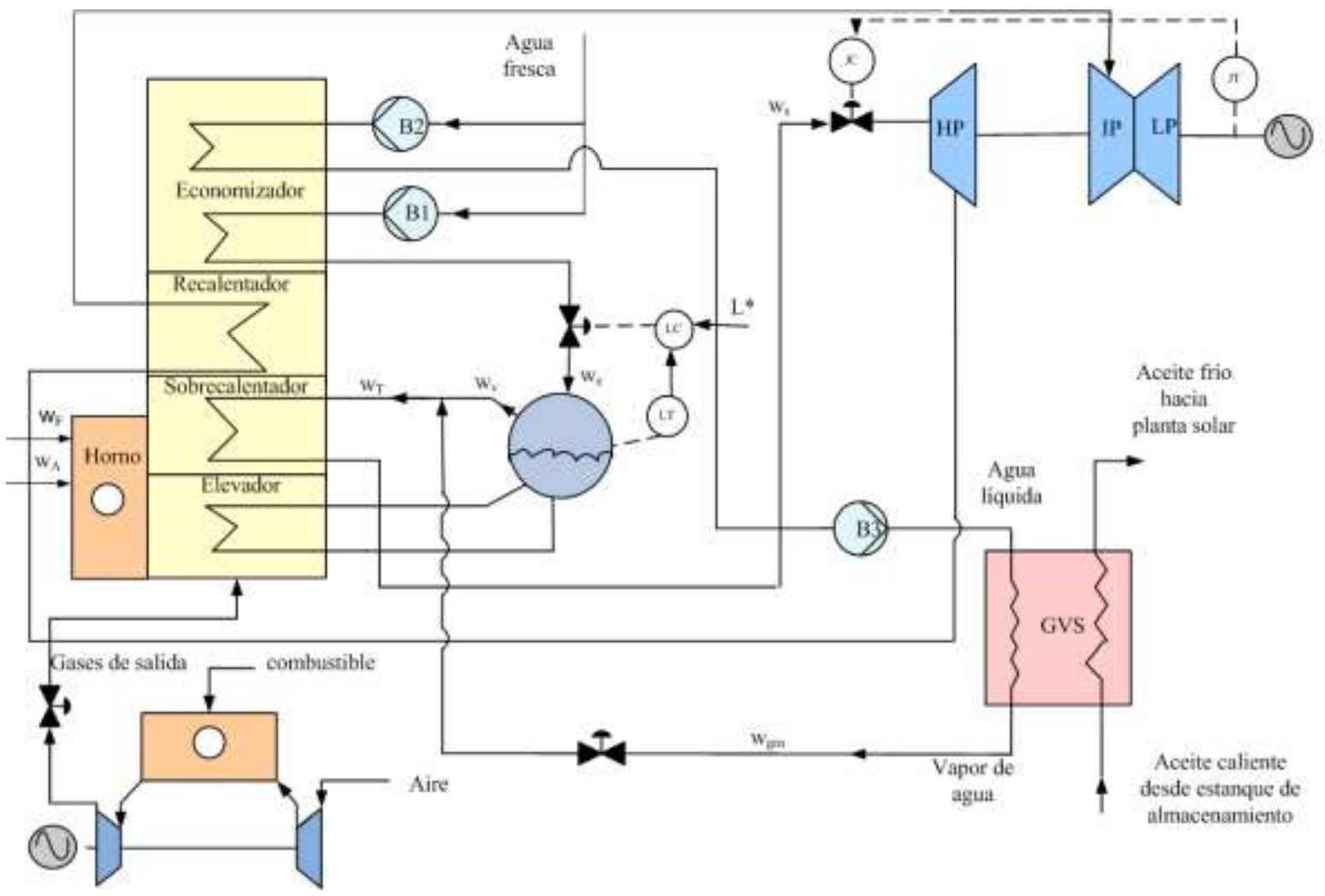

Fig. 2: Generador de vapor solar conectado a la planta de ciclo combinado.

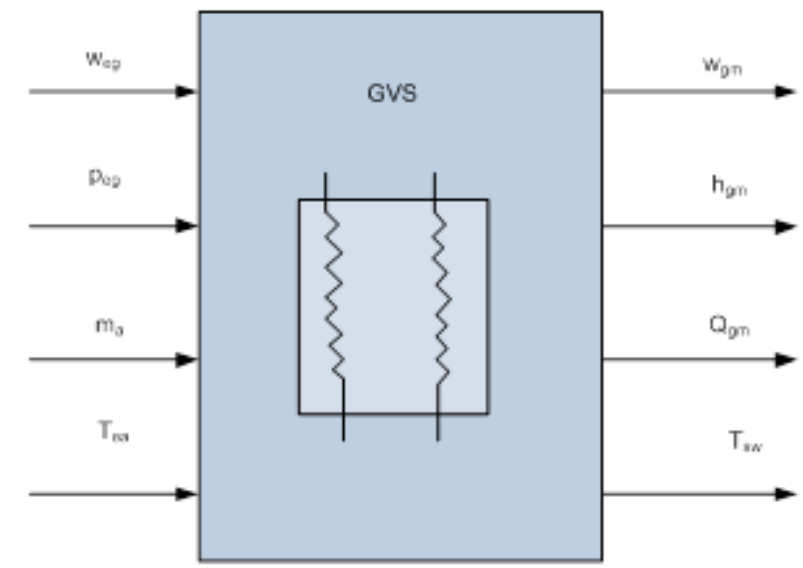

Fig. 3: Diagrama de entradas y salidas del generador de vapor solar

Tabla 1: Ecuaciones utilizadas en el diseño del simulador de GVS

\begin{tabular}{|c|l|l|l|}
\hline 1 & $\begin{array}{l}\text { Calor específico del aceite } \\
\text { Therminol 55 }\end{array}$ & $C_{p a}=1820+3.478 T_{a}$ & $\begin{array}{l}\text { Camacho } \\
\text { al.,(1993) }\end{array}$ \\
\hline 2 & Temperatura de saturación & $T_{v}=\frac{3816.4}{18.304-\ln \left(p_{e g}\right)}+46.13$ & Este trabajo \\
\hline 3 & $\begin{array}{l}\text { Entalpía del vapor saturado en } \\
\text { función de la temperatura }\end{array}$ & $\begin{array}{l}h_{g m}=-1.8934^{*} 10^{6}+4.1404^{*} 10^{4} T_{v}-148.7585^{*} T_{v}^{2} \\
+0.2471^{*} T_{v}^{3}-1.5519^{*} 10^{-4} T_{v}{ }^{4}\end{array}$ & Ordys et al., (1994) \\
\hline 4 & $\begin{array}{l}\text { Entalpía de vaporización en } \\
\text { función de la presión }\end{array}$ & $h_{f}=2.2464^{*} 10^{6}-0.0892 p_{g m}$ & Ordys et al., (1994) \\
\hline 5 & $\begin{array}{l}\text { Calor transferido al aceite por } \\
\text { radiación solar }\end{array}$ & $Q_{a}=m_{a} C_{p a}\left(T_{e a}-T_{s a}\right)$ & Este trabajo \\
\hline 6 & Calor de vaporización & $Q_{g m}=w_{e g} C_{l}\left(T_{v}-T_{e w}\right)+W_{e g} h_{f}+W_{e g} C_{g}\left(T_{s w}-T_{v}\right)$ & Este trabajo \\
\hline 7 & Balance de energía & $Q_{g m}=-Q_{a}$ & Este trabajo \\
\hline 8 & $\begin{array}{l}\text { Flujo de vapor (obtenido del } \\
\text { balance de energía) }\end{array}$ & $w_{g m}=Q_{a} /\left(C_{l}\left(T_{v}-T_{e w}\right)+h_{f}+C_{g}\left(T_{s w}-T_{v}\right)\right.$ & Este trabajo \\
\hline
\end{tabular}




\section{Programación del simulador}

Una vez definidas las ecuaciones que describen el generador de vapor solar, se implementa el programa utilizando la función s-function de Matlab Simulink®. Las funciones s-function son funciones programables por el usuario y que sirven para describir modelos dinámicos complicados. Para la simulación de sistemas dinámicos, existen unos ficheros plantilla cuyo propósito es ser copiados en el directorio de trabajo y particularizarlos con las instrucciones y parámetros del usuario. En la Figura 4 se muestra un diagrama de flujo del simulador del generador de vapor solar. Inicialmente se ingresan los valores de $T_{\text {ea }}$ y $m_{a}$ del aceite de la planta solar. También se ingresan el flujo de agua $w_{e g}$, la temperatura $T_{\text {ew }}$ y la presión $p_{e g}$, donde las dos primeras variables vienen del alimentador de agua de la planta de ciclo combinado y la segunda de la bomba instalada a la salida de dicho alimentador de agua. Se definen también los parámetros del generador de vapor, tales como volumen del GVS, la temperatura de entrada del agua y la capacidad calorífica del agua líquida y del agua vapor. Con los valores de las variables de entrada obtenidos, se resuelven las ecuaciones algebraicas desde la ecuación (1) a la (7) de la Tabla 1. Con esto se determina los valores de $h_{g m}, Q_{g m}$, cuyos valores ingresan al simulador del sobre calentador. De la ecuación (8) se obtiene el flujo de vapor producido $w_{g m}$. Esta operación se repite en cada tiempo de muestreo, como se indica con la línea punteada en la Figura 4.

\section{RESULTADOS}

El simulador desarrollado permite estudiar la evolución de variables relevantes del sistema, no solo ante la variación de la radiación solar, sino también ante la variación de otras variables. Para mostrar esto, se realizaron diversas simulaciones de forma de verificar el buen funcionamiento del simulador y apreciar algunas de sus aplicaciones. Para el diseño del simulador del generador de vapor solar GVS se consideró que la temperatura de entrada del aceite es constante, a pesar de que esta temperatura depende de la radiación solar disponible en la planta solar.

Sin embargo, la planta solar cuenta con un sistema de control de la temperatura del aceite lo que permite mantener esta temperatura constante, a pesar de las variaciones de la radiación solar durante el día. Para ello se varía el flujo de aceite de manera que la temperatura se mantenga en un valor especificado. Si la cantidad de radiación cambia (aumenta o disminuye) cambiará el flujo de aceite que llega al GVS. Cuando aumenta la radiación solar, aumenta el flujo de aceite de forma que la temperatura de entrada del aceite $T_{\text {ea }}$ al GVS se mantenga constante. Como se tiene mayor energía disponible, se produce un flujo $w_{e g}$ mayor de vapor sobrecalentado. Cuando la radiación solar disminuye también disminuye el flujo de aceite para que la temperatura de aceite se mantenga constante. Por lo tanto, disminuye también el flujo de vapor que va al sobre calentador.

Para mostrar el efecto que tiene la radiación solar sobre algunas variables de la planta ISCC, se hicieron simulaciones utilizando un perfil típico de radiación solar en función de la hora local como el mostrado en la Figura 5. En esta figura se aprecia que la hora de mayor radiación solar es a medio día y que disminuye por la tarde. Por la mañana la radiación solar es alrededor de $950 \mathrm{~W} / \mathrm{m}^{2}$, y aumenta a medida que se acerca el medio día. Cómo el simulador desarrollado es dinámico, es posible ver la evolución de las variables de la planta, cuando se le aplica este perfil de radiación.

La Figura 6 muestra la variación del flujo de aceite que entra al GVS desde la planta solar. El sistema de control de temperatura de la planta solar (Camacho et al., 1993) provoca que el flujo de aceite aumente en las horas de mayor radicación solar, lo que corresponde al medio día. A medida que disminuye la radiación el flujo de aceite disminuye, específicamente por la mañana antes de mediodía y por la tarde después de las 14 horas. El flujo de aceite debe variar entre 2 y $12 \mathrm{l} / \mathrm{s}$ por condiciones de diseño del simulador de la planta solar.

En la Figura 7 se muestra el flujo másico de vapor que produce el GVS, y que es inyectado al sobrecalentador en un día con el perfil de radiación dado en la Figura 5. Se aprecia que la mayor cantidad de flujo másico de vapor de salida del GVS se produce entre las 12 horas y las 14 horas. Esto coincide con la hora de mayor radiación solar y la mayor cantidad de flujo de aceite que entra al GVS. Después de las 14 horas la radiación solar disminuye, lo que provoca que el flujo de vapor que produce el GVS también disminuya. Por otro lado, si el aporte de vapor desde el GVS a la planta de ciclo combinado disminuye, el vapor producido en la planta de CC aumentará de manera de mantener constante el vapor que se inyecta a la turbina de vapor. 


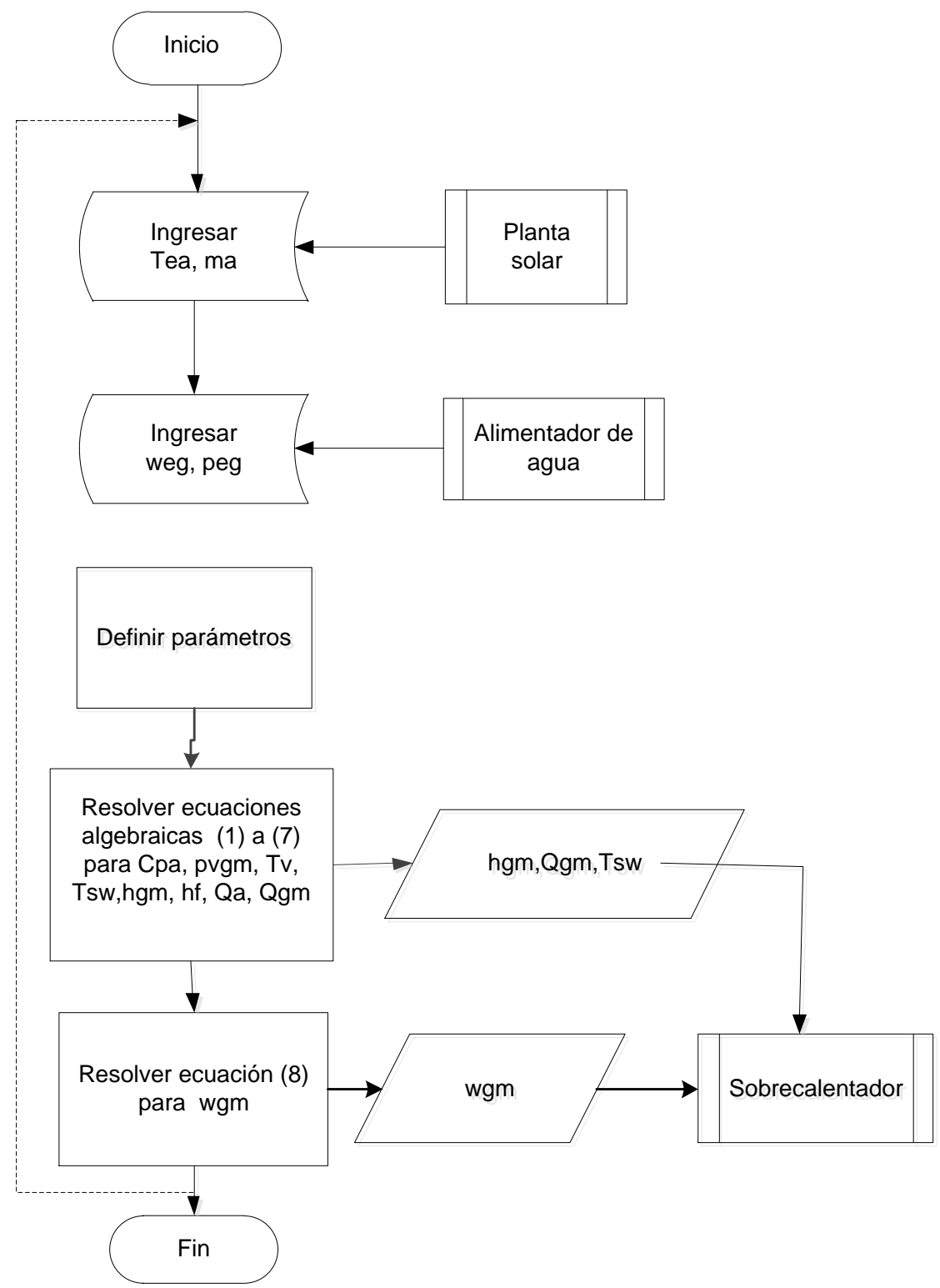

Fig. 4: Diagrama de flujo simulador generador de vapor solar

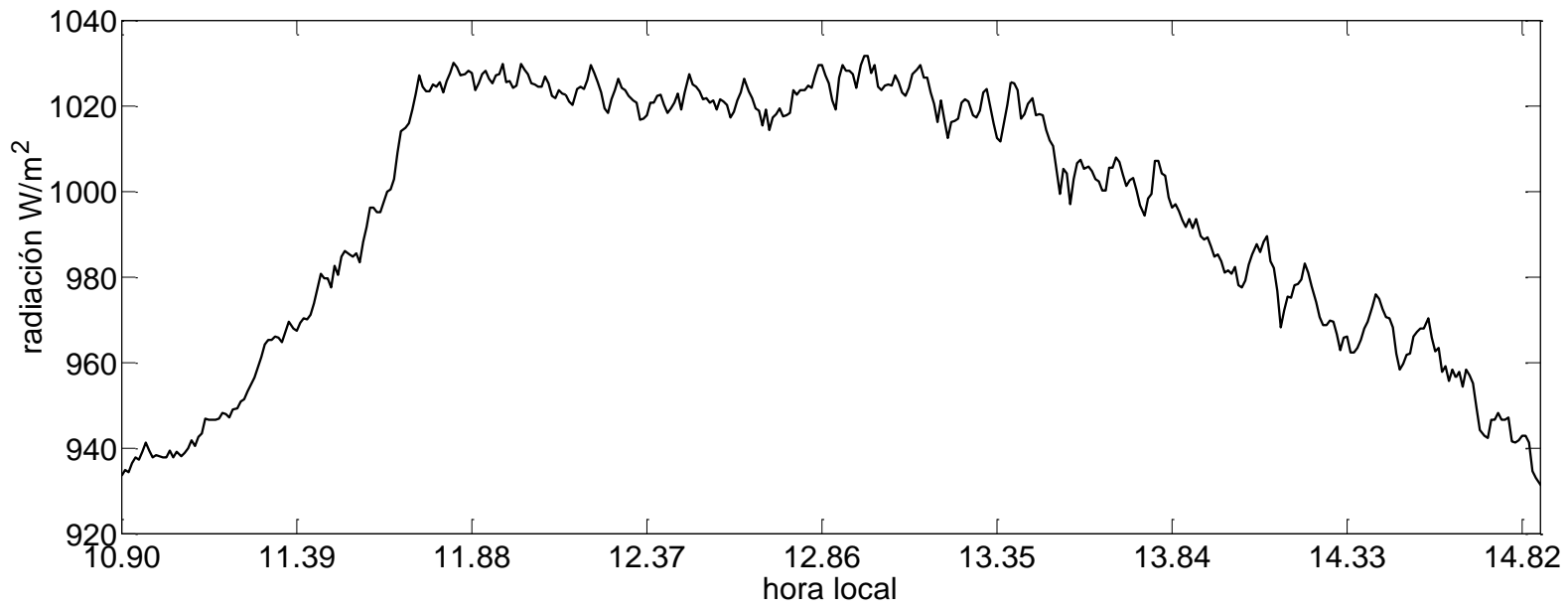

Fig. 5: Perfil de un día de radiación solar. (Camacho, et al.,1993) 


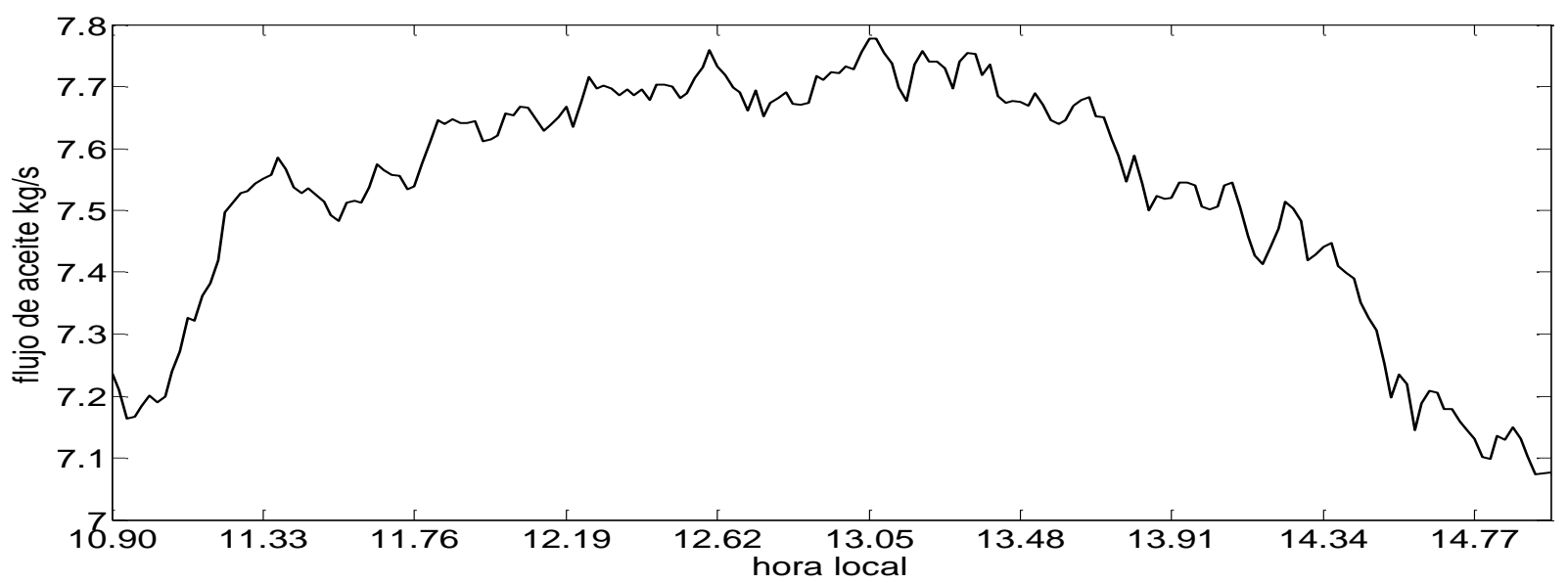

Fig. 6: Flujo de aceite cuando varía la radiación solar

En la Figura 8 se muestra cómo varia el calor suministrado por el quemador auxiliar al sobrecalentador a medida que cambia la radiación solar durante el día. La mayor cantidad de calor se produce en la mañana antes del mediodía y por la tarde después de las 14 horas. Al mediodía el calor suministrado al sobrecalentador por el quemador auxiliar disminuye, ya que en ese período de tiempo la cantidad de vapor producido por el GVS es mayor, debido a la mayor radiación solar disponible. Según lo recomendado por Kelly et al. (2001), una planta integrada será más económica que una planta solar para una contribución anual por sobre el $10 \%$ del vapor. En este estudio el porcentaje de contribución de vapor de la planta solar a la planta integrada (\%) se calcula en función del flujo de vapor $F_{P S}$ desde la planta solar y del flujo de vapor $F_{P C C}$ en la planta CC:

$$
\eta \%=\frac{100 * F_{P S}}{F_{P S}+F_{P C C}}
$$

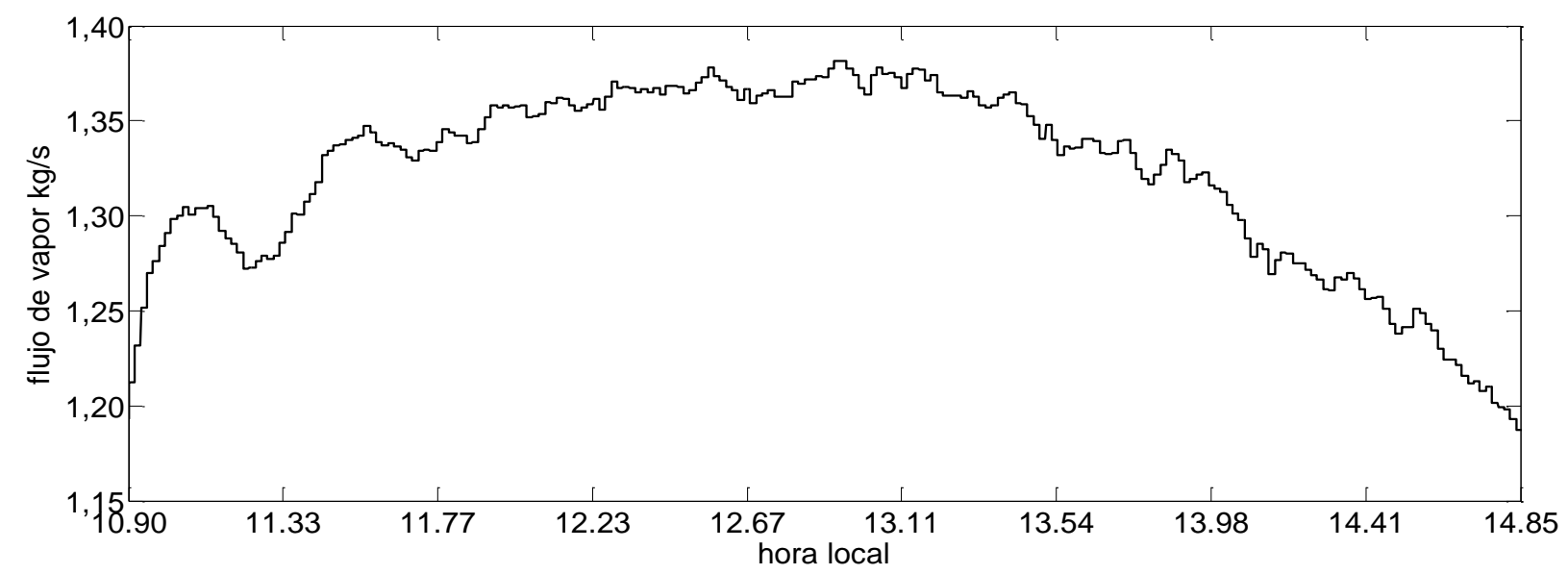

Fig. 7: Flujo de vapor producido por el GVS

Si se calcula la contribución solar a partir de los valores de la Figura 7, se tiene que el máximo aporte solar es de $10.1 \%$. Esto es considerando que el flujo de vapor producido por la planta de ciclo combinado es de $12(\mathrm{Kg} / \mathrm{s})$. Este valor se produce durante el período de mayor radiación solar, entre las 12 y las $13 \mathrm{~h}$. Por otro lado, la menor contribución solar se produce en la mañana a las $10 \mathrm{~h}$ y por la tarde a las $14 \mathrm{~h}$. En este caso la contribución es de un 9\%. Estos valores están dentro del rango propuesto por Kelly et al (2001).

\section{CONCLUSIONES}

De los resultados mostrados se pueden obtener las siguientes conclusiones principales: (i) El simulador desarrollado es una buena herramienta para estudiar los efectos de las variaciones de la radiación solar en una planta de ciclo combinado con colectores solares integrados; (ii) El simulador desarrollado permite observar la evolución de variables relevantes tal como el flujo de vapor que se produce en el GVS, calor suministrado por el quemador auxiliar y otras variables de la planta ISCC. 


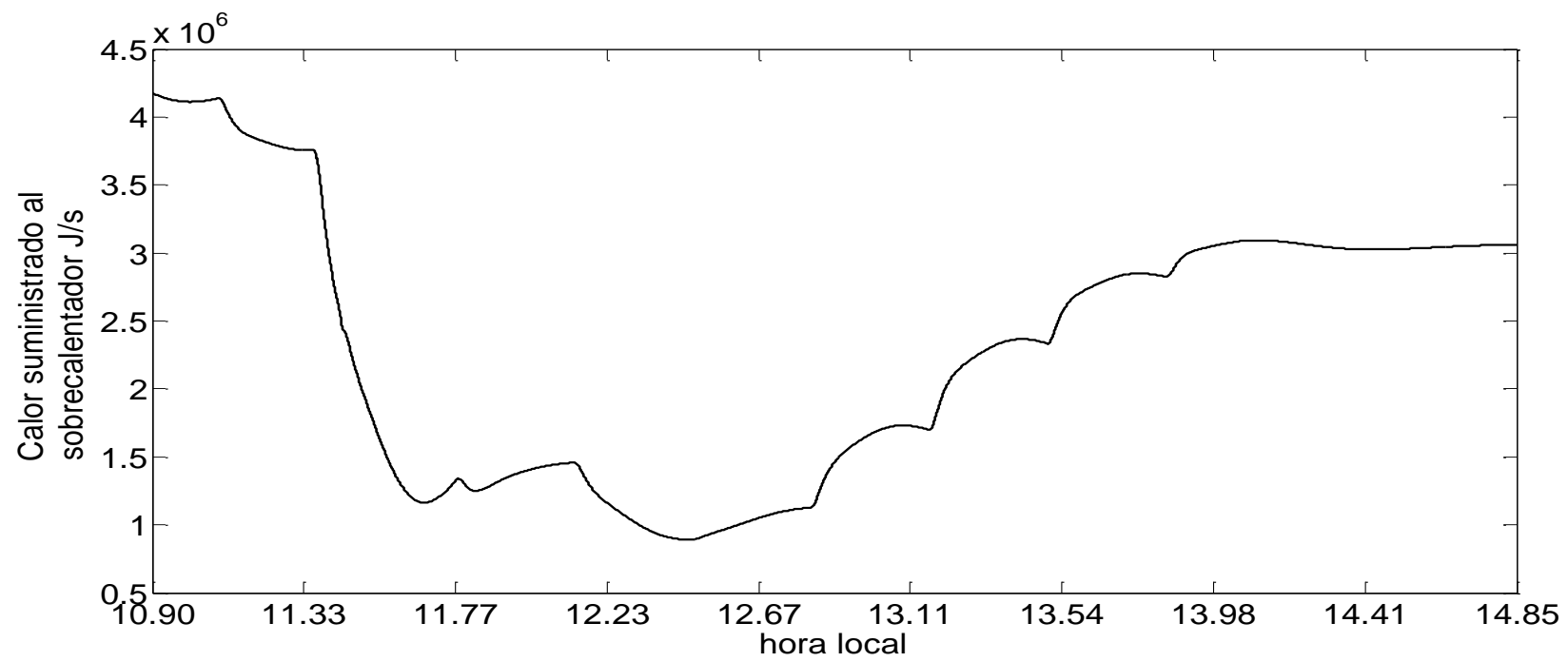

Fig. 8: Calor suministrado al sobrecalentador desde el horno auxiliar.

\section{NOTACION}

\begin{tabular}{|c|c|c|c|}
\hline Símbolo & Descripción & Valor & Unidades \\
\hline$C_{p a}$ & Calor específico del aceite & 3795.5 & $\mathrm{~J} /\left(\mathrm{kg}^{*} \mathrm{~K}\right)$ \\
\hline$C_{g}$ & Calor específico del agua vapor & 2260 & $\mathrm{~J} /\left(\mathrm{kg}^{\star} \mathrm{K}\right)$ \\
\hline$C_{l}$ & Calor específico del agua líquida & 4267 & $\mathrm{~J} /\left(\mathrm{kg}^{*} \mathrm{~K}\right)$ \\
\hline$h_{f}$ & Entalpía específica de evaporación & $1.8428 * 10^{6}$ & $\mathrm{~J} / \mathrm{kg}$ \\
\hline$h_{g m}$ & Entalpía específica del vapor del GVS & $2.8087^{*} 10^{6}$ & $\mathrm{~J} / \mathrm{kg}$ \\
\hline$m_{a}$ & Flujo másico de aceite del estanque de la planta solar & 3.6491 & $\mathrm{~kg} / \mathrm{s}$ \\
\hline$p_{e g}$ & Presión del flujo másico de entrada del agua del GVS & $21.75^{*} 10^{3}$ & $\mathrm{Mm} \mathrm{Hg}$ \\
\hline$Q_{a}$ & Calor suministrado al aceite desde la radiación solar & $2.7003^{*} 10^{6}$ & $\mathrm{~J} / \mathrm{s}$ \\
\hline$Q_{g m}$ & Calor suministrado al vapor del GVS & $2.7003^{*} 10^{6}$ & $\mathrm{~J} / \mathrm{s}$ \\
\hline$T_{e a}$ & Temperatura de entrada del aceite del estanque de la planta solar & 568 & $\mathrm{~K}$ \\
\hline$T_{s a}$ & Temperatura de salida del aceite del GVS & 450 & $\mathrm{~K}$ \\
\hline$T_{v}$ & Temperatura de saturación de vapor & 505 & $\mathrm{~K}$ \\
\hline$T_{\text {ew }}$ & Temperatura de entrada del agua líquida & 408 & $\mathrm{~K}$ \\
\hline$T_{s w}$ & Temperatura de salida del vapor sobrecalentado & 558 & $\mathrm{~K}$ \\
\hline$w_{g m}$ & Flujo másico de vapor desde GVS & 1.2 & $\mathrm{~kg} / \mathrm{s}$ \\
\hline$w_{e g}$ & Flujo másico de entrada del agua líquida & 1.2 & $\mathrm{~kg} / \mathrm{s}$ \\
\hline$\eta$ & Contribución de vapor & & \\
\hline \multicolumn{4}{|c|}{ Abreviaturas } \\
\hline $\mathrm{CC}$ & Ciclo combinado & & \\
\hline DSC & Colectores solares distribuidos & & \\
\hline $\mathrm{F}_{\mathrm{PCC}}$ & Flujo de vapor desde la planta de ciclo combinado & & \\
\hline FPS & Flujo de vapor desde la planta solar & & \\
\hline GEI & Gases Efecto Invernadero & & \\
\hline GVS & Generador de Vapor Solar & & \\
\hline HRSG & Caldera recuperadora de vapor & & \\
\hline HTF & Fluido de transferencia de calor & & \\
\hline ISCC & Ciclo combinado con colectores solares integrados & & \\
\hline
\end{tabular}

\section{AGRADECIMIENTOS}

Los autores agradecen a la Dirección de Investigación de la Universidad de La Serena (Chile) por su apoyo a través del proyecto PI15351. 


\section{REFERENCIAS}

Aftzoglou, Z." Exploring Integration Options in the Energy Sector, Including a Case Study of the Integration of Solar Thermal Energy into a Combined Cycle Power Plant', Tesis de Magister, Delf University of Technology, Holanda (2011)

Behar, O., Kellaf, A., Mohamedi, K. y Belhamel, M., "Instantaneous Performance of the First Integrated Solar Combined Cycle System in Argelia", Energy Proceeding, 6, 185-193 (2011)

Camacho, E., Berenguel, M. y Rubio, F., "Simulation Software Package of the Acurex Field", Sevilla: E.S.I. of Sevilla, Internal Report, (1993)

Central Energía, Central Energía. Central de información y discusión de energía en Chile. (En línea: http://www.centralenergia.cl/2010/11/18/crecimiento-economico-y-abastecimiento-energetico/_acceso: 20 de Mayo, 2014 (2010)

Dersch, J., Geyerb, M. y otros siete autores, "Trough Integration into Power Plants- a Study on the Performance and Economy of Integrated Solar Combined Cycle System", Energy, 29, 947-95, (2004)

Greenpeace, "Energía y Cambio Climático", (en línea: http://www.greenpeace.org/ mexico/es/Campanas/ Energia-y-cambio-climatico/, acceso: 13 Mayo, 2014 (2014)

Horn, M., Führing, H. y Rheinländer, J., "Economical Analysis of Integrated Solar Combined Cycle Power Plants. The Economic Feasibility of an ISCCS Power Plant in Egypt”, Energy, 29, 935-945 (2004).

Hosseini, R., Soltani, M. y Valizadeh, G., "Technical and Economic Assessment of the Integrated Solar Combined Cycle Power Plants in Iran", Renewable Energy, 30, 1514-1555 (2005)

Kelly, B. y Herrmann, U., "Integrated Solar Combined Cycle Systems", National Renewable Energy Laboratory", [En línea]: http://www.nrel.gov/csp/troughnet/pdfs/kelly_isccs_analysis.pdf, acceso: 6 de Abril, $2013(2001)$

Kelly, B., Herrmann, U. y Hale, M., "Optimization Studies for Integrated Solar Combined Cycle Systems", Washington, Proceeding of Solar Forum. Solar Energy: The Power to Choose, (2001)

Ordys, A., Pike, A., Johnson, M. y Katebi, R., "Modelling and Simulation of Power Generation Plants", Primera ed. London: Springer-Verlag, (1994)

Sáez, D., Cipriano, A. y Ordys, A., "Optimization of Industrial Processes at Supervisory Level. Application to Control of Thermal Power Plants", Primera ed. London: Springer-Verlag, (2002) 